Andreas Pysiewicz, Stefan Weinzierl

\title{
Instruments for Spatial Sound Control in Real Time Music Performances. A Review
}

Chapter in book | Accepted manuscript (Postprint)

This version is available at https://doi.org/10.14279/depositonce-9008

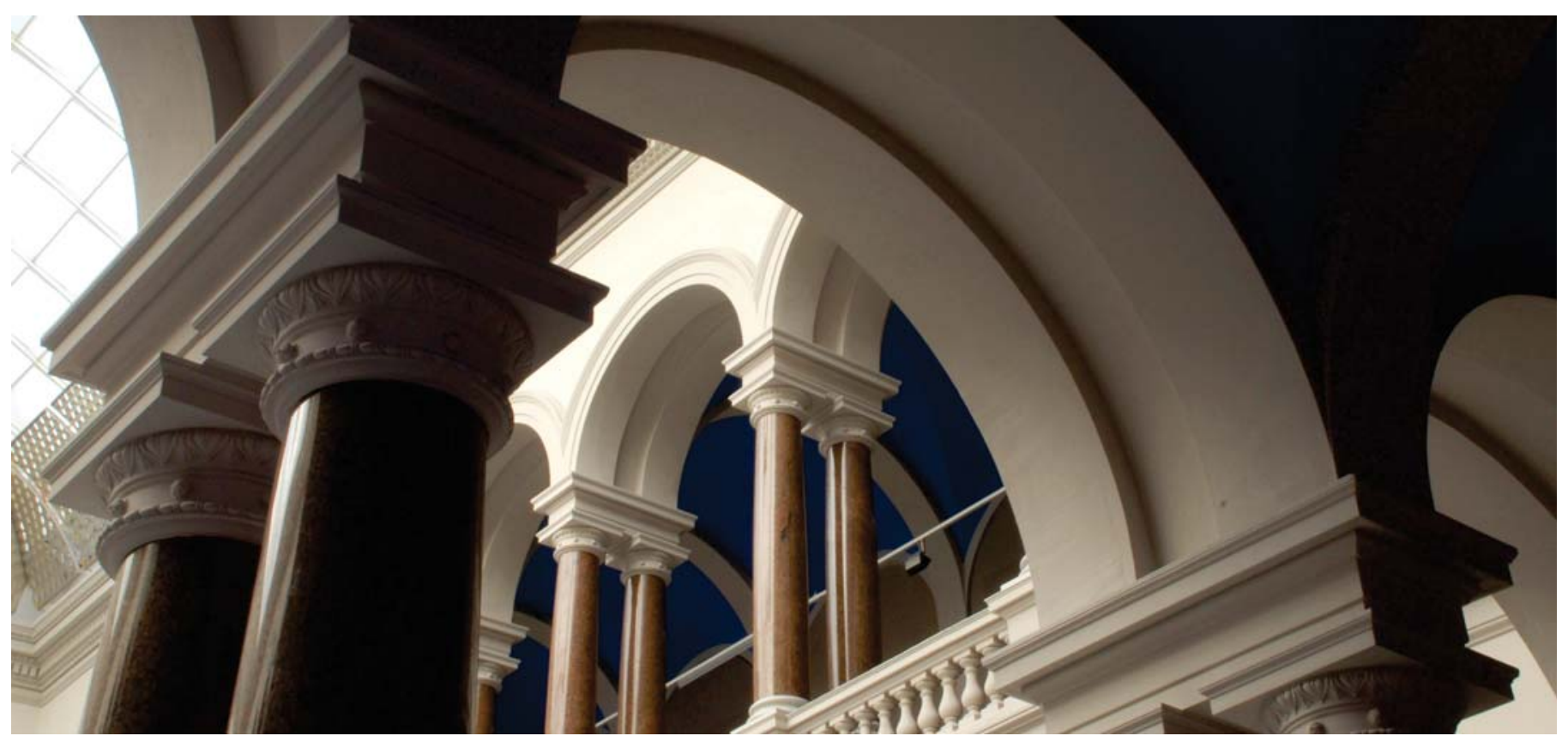

Pysiewicz, A., \& Weinzierl, S. (2016). Instruments for Spatial Sound Control in Real Time Music

Performances. A Review. In: Bovermann et al. (Eds.): Musical Instruments in the 21st Century (pp. 273-

296). Springer Singapore. https://doi.org/10.1007/978-981-10-2951-6_18 


\title{
Instruments for Spatial Sound Control in Real Time Music Performances. A Review
}

\author{
Andreas Pysiewicz and Stefan Weinzierl
}

\begin{abstract}
The systematic arrangement of sound in space is widely considered as one important compositional design category of Western art music and acoustic media art in the 20th century. A lot of attention has been paid to the artistic concepts of sound in space and its reproduction through loudspeaker systems. Much less attention has been attracted by live-interactive practices and tools for spatialisation as performance practice. As a contribution to this topic, the current study has conducted an inventory of controllers for the real time spatialisation of sound as part of musical performances, and classified them both along different interface paradigms and according to their scope of spatial control. By means of a literature study, we were able to identify 31 different spatialisation interfaces presented to the public in context of artistic performances or at relevant conferences on the subject. Considering that only a small proportion of these interfaces combines spatialisation and sound production, it seems that in most cases the projection of sound in space is not delegated to a musical performer but regarded as a compositional problem or as a separate performative dimension. With the exception of the mixing desk and its fader board paradigm as used for the performance of acousmatic music with loudspeaker orchestras, all devices are individual design solutions developed for a specific artistic context. We conclude that, if controllers for sound spatialisation were supposed to be perceived as musical instruments in a narrow sense, meeting certain aspects of instrumentality, immediacy, liveness, and learnability, new design strategies would be required.
\end{abstract}

A. Pysiewicz (corresponding author), S. Weinzierl

Audio Communication Group, Technische Universität Berlin, Berlin, Germany

e-mail: andreas.pysiewicz@tu-berlin.de

S. Weinzierl

e-mail: stefan.weinzierl@tu-berlin.de 


\section{Introduction}

The arrangement of sound in space as an integral part of the musical composition can be observed as a central concern of current art music practice. In the field of electronic and electroacoustic music in particular, one will hardly find musical performances which do not make systematical use of multiple loudspeaker projection techniques or site-specific spatial arrangements. Along with the description of different artistic concepts related to the organization of sound in space (e.g., Stockhausen 1959; Leitner 1971; Xenakis 1992), also the techniques for sound spatialisation have been addressed with respect to a formalization and classification of their artistic and technical principles within the last decades (e.g., Roads 1996; Zvonar 2000; Baalman 2010; Sannicandro 2014).

While the critical examination of space as a musical parameter is not a general novelty, increasing attention has been paid to different aspects of real time sound spatialisation as performance practice. This observation is indicated by a growing number of concepts and instruments that have been developed to explore performative degrees of freedom for the spatial presentation of music, primarily introduced by communities in the context of the New Interfaces of Musical Expression (NIME), the International Computer Music Conference (ICMC) or the Sound and Music Computing (SMC) conferences. Understanding space as a significant musical parameter (alongside with pitch, timbre, intensity and duration), it is only consistent that spatial sound controllers are regarded as specific instruments of musical expression, too.

Beyond these general considerations, however, there is a lack of systematic approaches to identify, analyse and contextualize real time spatial sound controllers in the interplay of musical expression, artistic performance practice, audio technologies, and the field of human-computer interaction (HCI), as represented by the theory of digital musical instruments (DMI).

In the following, we will use this broader perspective and attempt to provide a structured review of instruments and interface concepts introduced to enable performers to control spatial parameters from the early days of electronic music performances to date. After a brief introduction to the terminological and methodological framework used to categorize a collection of spatial controllers, we develop a systematic taxonomy, by which we obtain a clearer, theory-based perspective on current trends of interface design in the domain of musical expression and provide an outlook on conceptual consequences for potential future developments.

\section{Defining the Contexts: Space and Spatialisation of Sound}

Along with the use of technology to expand musical boundaries, the notion of space as a musical parameter is considered as one constitutive element of electroacoustic music. Unlike with other parameters such as timbre or pitch, it is challenging to 
define the notion of space precisely, since it can refer to a multitude of phenomena ranging from architecture, room acoustics and spatial hearing to conceptual and metaphorical uses, such as the tonal space or structural concepts of musical foreground and background.

To avoid any terminological confusion, we will here refer to sound spatialisation to indicate a group of techniques for organizing and manipulating the spatial projection and movement of sound in a physical or virtual listening environment (Valiquet 2011). ${ }^{1}$ Similarly, the term (electroacoustic) sound diffusion, frequently used synonymously for sound spatialisation, refers to the real time distribution of sound throughout space by controlling the relative levels, equalization and localization of sound during performance. While often related to the performance practice of fixed media compositions (stereo or multi-channel), the source material may also be generated live. ${ }^{2}$

Roads (1996, 451) emphasizes the duality of physical and perceptual layers within the act of sound spatialisation and states the dramaturgic and structural importance of spatial movements. Thus, not only the technical developments, but also the knowledge of mechanisms and cues for spatial perception, as it was acquired throughout the 20th century, is crucial for the application of spatial sound projection techniques. Refer to Brech (2015) for a historical review on the examination and musical operationalization of spatial perception, with Roads (1996) and Blauert (1997) providing comprehensive insights on the relevant psychoacoustic phenomena.

When investigating on the role of spatialisation in compositional and performance practice of Western art music, one will find space-related techniques throughout musical genres and eras such as early antiphonic choral writings or specific orchestral techniques reaching back to the 16th century. However, spatialisation was not generally considered a crucial parameter of musical expression before the advent of electroacoustic music being obviously related to the availability of appropriate technical resources. With new technical means, composers started to think about spatial organization in their pieces in a very different way, involving new forms of spatial aesthetics. Many composers quickly adapted their musical concepts to the new (spatial) techniques resulting in a demand for new and better technologies to realize their refined spatialisation conceptions. As a consequence, technological advance in the last two decades of the 20th century, the rise of digital production technologies and the increasing efficiency of spatial rendering algorithms, especially methods of sound field synthesis (wave field synthesis or

\footnotetext{
${ }^{1}$ Accordingly, the term spatial music was coined to highlight electroacoustic compositions in which the dynamic projection of sound sources is an integral part of compositional process. While the practice of spatialisation can be applied to any kind of spatial sound projection, it mainly refers to the field of electroacoustic music.

${ }^{2}$ Sound diffusion is originally used for the live presentation of acousmatic music, a form of electroacoustic music composed for (multiples of) loudspeakers using recorded sound material out of their original context. Interestingly, sound diffusion as performance practice is conceptually related to one specific control interface: the fader board of mixing desks (see our taxonomy).
} 
higher order ambisonics, to mention only two important techniques) has greatly affected (and still affects) performance practice of electroacoustic music.

\subsection{Sound Spatialisation as Performance Practice}

With respect to the concepts and tools for sound spatialisation, different systematic approaches have been developed. Malham (1998) outlines three basic techniques of sound spatialisation: binaural reproduction of the sound field (by providing the signals directly at the ears), stereophonic sound projections by means of loudspeaker orchestras (a.k.a. sound diffusion) and sound field synthesis techniques (e.g., by means of ambisonic systems). ${ }^{3}$ Whereas binaural techniques do not play a noteworthy role for performance practice, the latter two can be considered as well established paradigms of realtime spatialisation. ${ }^{4}$

Following Lynch and Sazdov (2011) who differentiate between three artistic concepts of spatialisation in electroacoustic music we can categorize three main approaches of sound spatialisation considering all possible means:

- Sound spatialisation based on properties of the fixed audio material (mainly related to the spectral $^{5}$ or temporal features).

- Sound spatialisation based on algorithmic or stochastic ${ }^{6}$ processes (not related to the analysis of the audio material), controlling the spatial presentation without direct access to spatial parameters.

- Sound spatialisation based on the direct access to the spatial projection of the sounds and/or manipulation of their properties in real time (by means of decorrelation, panning, or more complex methods of sound field reproduction). ${ }^{7}$

The major distinction between these categories lies in the degree of active control, the performer can exercise over the spatialisation process during the performance. While the spatialisation within the first two categories is highly determined by either the texture of the material itself or the algorithm in use, the last

\footnotetext{
${ }^{3}$ Since we cannot address the technical principles of sound field synthesis here, the reader can refer to Geier et al. (2010) for further details on wave field synthesis, ambisonics techniques and recent stereophonic panning methods.

${ }^{4}$ The transition from amplitude panning techniques to methods of sound field synthesis represents a paradigm shift of sound spatialisation (Geier et al. 2010): from a channel-based approach (controlling a single channel assigned to one loudspeaker) to an object-based approach (controlling a sound object in space).

${ }^{5}$ For a comprehensive review of spectral spatialisation techniques, see Jaroszewicz (2015).

${ }^{6}$ It might seem paradox to include stochastic processes to a category mainly defined by determined characteristics, however they are grouped here due to their decreased realtime controllability in terms of exact spatial deployment.

${ }^{7}$ This category may also include mapping strategies in which the synthesis process of the sound material directly affects its spatialisation, in contrast to the static spatialisation process of fixed audio material in the first category.
} 
category offers a direct mapping of controller data to spatial parameters. All these types of spatialisation can generally be applied during performance, yet the latter paradigm represents the most common approach to sound spatialisation to date. Correspondingly, Baalman (2010) describes exclusively spatial techniques that are based on the direct manipulation of spatial parameters ranging from control over location and trajectories of sound sources to more complex parameters such as enhanced acoustic characteristics of the space. Having identified some major concerns of spatialisation practice, in the following we take a look at the distinctions different concepts and implementations of common sound spatialisation systems. It is important to note that the terms spatialisation controller, interface, instrument or system are used inconsistently and interchangeably in relevant literature, most commonly denoting an (electronic) apparatus to control the spatial behavior of sound. While controller, interface or instrument frequently refer to the specific device the performer is operating, the spatialisation system often means the set of (digital) components to render audio streams for the spatialisation process.

\subsection{Sound Spatialisation Controllers in Context of Digital Musical Instruments}

As outlined above, spatialisation can be considered as established artistic practice in the broader field of electroacoustic music and live electronic music. We have discussed that prevalent spatial sound techniques can generally be applied both to the production process (mainly in the studio) and to the real time presentation of music in the respective performance space. Zvonar (2000) formally differentiates between the live performance approach to sound spatialisation and techniques for pre-composed spatial arrangements of sound, such as environmental multichannel soundscape, classic studio-based multi-track composition and automated spatial control. Accordingly, only some available implementations of sound spatialisation systems are suited for use during performance. While several spatialisation controllers have been designed explicitly as studio production means, other system designs have simply met the limits of contemporary technologies, be it in terms of computational power for spatial rendering or the lack of suitable control interfaces. ${ }^{8}$

Furthermore, the complexity of the control task can be considered as another substantial obstacle for real time spatialisation. Such control and mapping related issues are well-known and dealt with in the field of human-computer interaction (HCI) and especially in the interaction design for interfaces of musical expression, an applied subfield of HCI. Therefore, it seems reasonable to regard the means for

${ }^{8}$ One can consider Stockhausen's Rotationstisch (a loudspeaker mounted to a rotating turntable system) as typical tool for spatial studio composition (Brech 2015). The spatialisation system used by Chowning to realize his simulation of moving sound sources (Chowning 1971) represents a typical studio approach. Simultaneously, it was clearly limited by processing performance of the 1970s (Zvonar 2000). 
spatial sound control used in live musical performance from the perspective of design practice of digital musical instruments (DMIs). This potential link has already been roughly explored in previous research (Wanderley and Orio 2002; Marshall et al. 2007; Schacher 2007; Perez-Lopez 2015), with a particular focus on the gestural control paradigm. At the core of the DMI metaphor, as introduced by Miranda and Wanderley (2006), stands the decoupling of the physical interface (input or control device) from the sound generating system (contrasting to the integral concept of acoustic musical instruments). Both instances are connected via a mapping layer assigning outputs of the controller to the inputs of the sound rendering engine. This modularization offers new degrees of freedom for the instrument design, however, the alleged decorrelation between the physical action of the performer and the produced sound, raises new issues related to the appreciation of the artistic performance (cf. Emerson and Egermann, this volume).

Considerations on both the control interface and the mapping structure are crucial for the instrument design in order to minimize control complexity without limiting its functionality. For a systematic outline of mapping strategies refer to Miranda and Wanderley (2006). Marshall et al. (2007) discuss common control issues and introduce three levels of spatial sound control parameters which are related to (1) the position, orientation and movement of the sound source and sink, respectively, (2) characteristics of the sound source (and sink), and (3) environmental and room model parameters (Marshall et al. 2007, 229). For a list of typical parameters related to all three levels see Table 1.

Beyond the aforementioned control aspects, the interaction interface includes the feedback side - be it visual, auditory, or tactile-kinaesthetic feedback-primarily experienced through the physical device itself and secondarily as an intended (auditory) result of the sound generation process (Miranda and Wanderley 2006, 11).

In order to compare and analyse musical interfaces appropriately, different classification systems have been developed, the most common one going back to Miranda and Wanderley (2006). Based on the resemblance to existing musical

Table 1 Spatialisation system control parameters (based on Marshall et al. 2007; Perez-Lopez 2015)

\begin{tabular}{l|l|l}
\hline $\begin{array}{l}\text { Sound source } \\
\text { orientation }\end{array}$ & $\begin{array}{l}\text { Sound source } \\
\text { characteristics }^{\mathrm{a}}\end{array}$ & $\begin{array}{l}\text { Environmental/room } \\
\text { parameters }\end{array}$ \\
\hline Position $(\mathrm{X}, \mathrm{Y}, \mathrm{Z})$ & Size & Size \\
\hline Elevation & Directivity & Presence \\
\hline (Trajectories) & Presence/distance & Early reflections \\
\hline & Brilliance/warmth & Reverberation \\
\hline & & Reverb. Cut-off Freq. \\
\hline & & Doppler effect \\
\hline & & Air absorption \\
\hline & & Equalization \\
\hline & & Geometry \\
\hline
\end{tabular}

${ }^{a}$ Parameters refer to sound source and sink respectively 
instruments, the authors distinguish between augmented musical instruments, instrument-like or instrument-inspired controllers, and alternate controllers. Especially the category of alternate controllers - subsuming various different interface concepts beyond the physical-mechanical interaction paradigm of acoustical instruments - can be broken down into sub-categories related to their sensing functionality relative to the human (Paradiso 1997; Mulder 2000): touch controllers react on direct physical manipulation (like a button or knob); non-contact or expanded-range controllers provide a limited sensing range for control gestures without physical contact (e.g. by using an infrared sensor system). Wearable or immersive controllers capture the control gestures with few or no restrictions to the movement since the performer is always in the sensing field (either by using, e.g., a sensor glove, suit or wide-range camera tracking system).

A special form of wearable controller can be found in biofeedback interfaces allowing for the acquisition of electrical signals generated by the human's muscles, eyes, heart or brain. Although present for over 50 years now in the field of music and interactive media art, these interfaces have played no significant role as spatial performance instruments, most likely due to the limited controllability and bandwidth of some of the captured parameters (such as brain waves). ${ }^{9}$

For a larger subgroup of alternate controllers, Overholt (2011) uses the term borrowed controller in order to emphasize that these have not originally been designed as a musical interface, such as video game controllers, camera tracking systems, etc. Interestingly, most spatialisation controllers can be assigned to this category.

Related to the control paradigm, further criteria to distinguish between different realizations of spatialisation controllers can be addressed. With respect to DMIs, Pressing $(1990,14)$ and Birnbaum et al. (2005, 193-94) propose multidimensional description spaces dealing with different aspects related to the controller and its relation to both the performance and the performer. Perez-Lopez (2015) derives a set of dimensions relevant for the analysis of spatialisation systems, including:

- Role of the performer - the performer exclusively controls spatial parameters in contrast to a performer who controls both spatialisation and sound synthesis.

- Required user competency - casual untrained users in contrast to trained expert users aiming at expressivity and virtuosity.

- Number of performers - most spatialisation instruments have been designed for a single performer; however, the control task could also be (functionally) shared by a group of performers.

\footnotetext{
${ }^{9}$ There is consensus that Music for Solo Performer (1965) by Alvin Lucier, scored for "enormously amplified brainwaves and percussion", was the first composition to make use of a biofeedback interface to control percussion instruments by the resonance of the performers brain activity (Miranda and Wanderley 2006). Several further artistic experiments have followed using biofeedback interfaces. Refer to Miranda and Castet (2014) for a comprehensive review on brain related interfaces.
} 
- Multiplicity of control-denotes the relationship between the quantity of simultaneous control streams available and the requirement to control these parameters continuously (as opposed to a default state when no control signal is present).

- Control Monitoring — related to the real time feedback modalities provided by the system on the executed control (e.g. by using a graphical user interface).

Having discussed the premises of spatialisation as performance practice in the field of electroacoustic music and contextualized real time spatialisation controllers within the discourse of DMIs and HCIs, we will provide a systematic inventory of spatialisation controllers presented to the public from the 1950 s till today in the following.

\section{A Systematic Inventory of Spatial Sound Controllers for Real Time Performance}

There have been a few recent attempts to review the evolution of spatialisation controllers from a historical and musicological perspective (e.g., Brech 2015; Brech and Paland 2015). Some authors have explicitly focused on spatialisation interfaces for real time performances of music (Mooney 2005; Johnson et al. 2013, 2014a, b), others have discussed more recent developments of sound spatialisation systems and spatial rendering frameworks (Marshall et al. 2007; Perez-Lopez 2015; Peters 2011; Peters et al. 2009; Schacher 2007) as the core component of common software solutions for sound spatialisation.

By providing a classification system and a first systematic inventory of spatialisation controllers, our contribution aims at providing deeper insight into design and performance practice of spatial sound controller. In order to guide future design efforts, we intend to gain a better understanding about the concepts that led to the specific developments for sound spatialisation practice.

\subsection{Study Design and Methodology}

Having outlined common categories and dimension spaces related to DMIs and their adaptions to spatialisation instruments, we suggest three dimensions for a taxonomy of spatialisation controllers (Fig. 1).

The first dimension is derived from the extended DMI taxonomy adopted by Miranda and Wanderley (2006) and Mulder (2000), which has been discussed above. As outlined before, due to conceptual similarities of spatialisation interfaces for real-time performances and digital musical instruments, we consider it reasonable to classify the controllers under the terms of musical instruments. However, since spatialisation instruments are rarely directly derived from traditional musical 


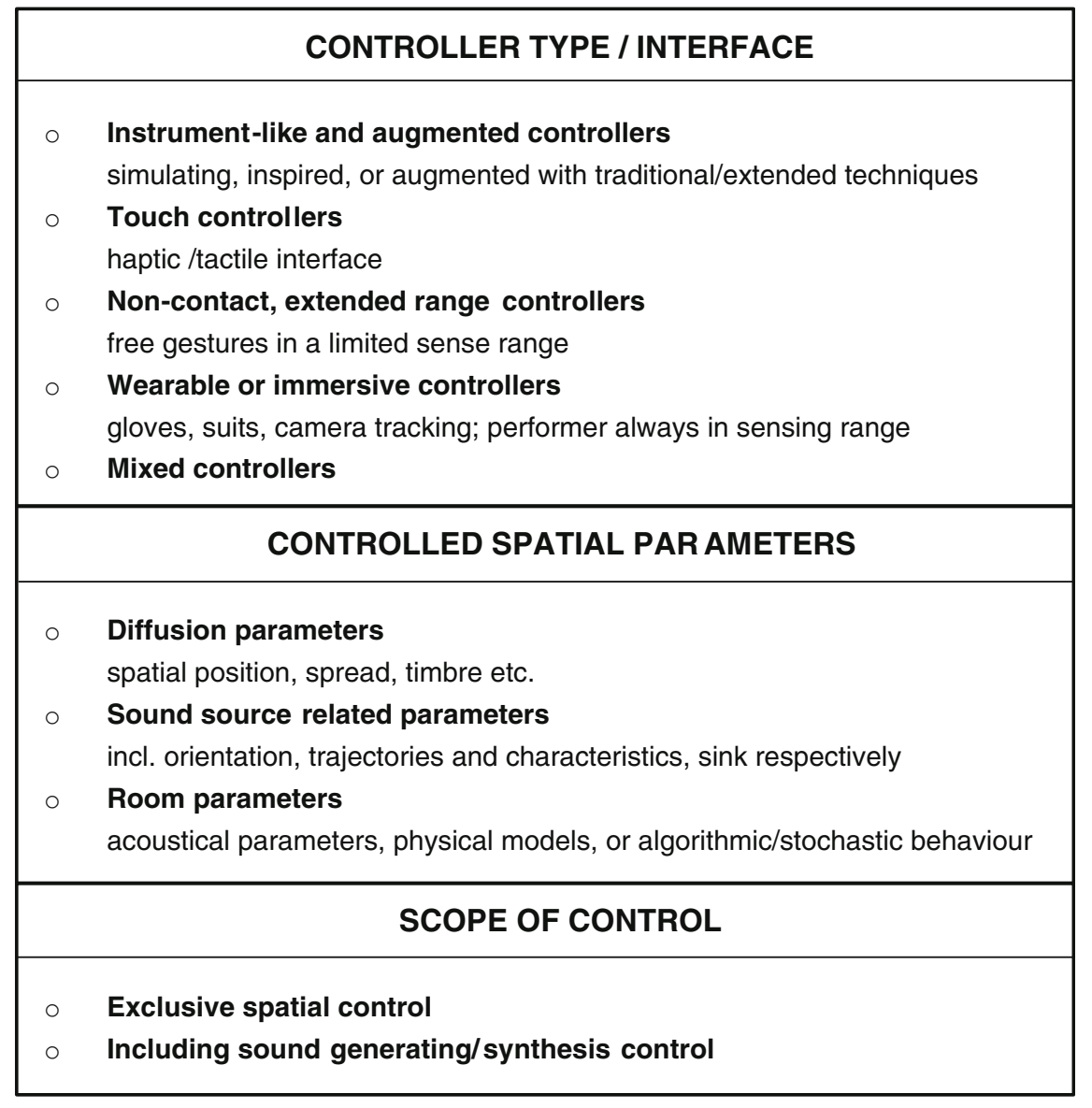

Fig. 1 Dimension space for the classification of spatialisation controllers

instruments, ${ }^{10}$ we have customized the set by combining some categories and adding another category (a mixed set of multiple sensors combining different categories) common for the practice of spatialisation. Since the category of alternate controllers tend to be the rule rather than the exception, we will not use the term alternate and instead directly refer to the control interface paradigm these instruments follow.

The second dimension refers to the scope of spatial parameters, which are controlled by the performer in real time using the spatialisation interface (see

\footnotetext{
${ }^{10}$ It remains a matter of ongoing discourse, whether certain kinds of production or reproduction devices (the record player or a mixing desk, for instance) can be considered as musical instruments. See Hardjowirogo (this volume), for a thorough discussion of musical instrument identity issues.
} 
previous section). Similar to the first dimension above, we have adapted the categories to embrace all relevant parameters ordered according to their complexity. Here, we decided to distinguish explicitly between diffusion parameters, i.e. parameters controlled by a channel-based diffusion system such as sound presence, position, or spread of the sound image, in contrast to spatial parameters of a sound source related to an object-based spatialisation approach. Since we could identify only a small number of complex spatial parameters in our explored data (such as room or environmental parameters, extended physical models or spatialisation algorithms), we created a shared category for all remaining parameters.

Referring to the role of the performer and the scope of integrated control options, the last dimension differentiates between instruments to control the spatial sound projection exclusively and instruments designed to handle the sound synthesis process as well. This category is closely related to the general concept of the instrument being either explicitly a spatialisation instrument or a self-contained music instrument with additional means for the spatial sound projection. In the latter case, we would expect a high correlation with the augmented instrument category in the first dimension of our taxonomy.

\subsubsection{Research Focus, Limitations and Resources}

As outlined before, we aimed at gathering data on all kinds of real time spatialisation instruments used in the history of western art music practice ranging from early developments in the middle of the $20^{\text {th }}$ century to present implementations. The most important prerequisite for the spatialisation controller to be included in our survey was their potential to be used in real time as part of an artistic performance. Thereby, it was irrelevant whether the spatialisation instrument has been presented only once in a single performance or artistic demonstration or whether it has gained a certain popularity for spatialisation practice.

Another conceptual requirement was the existence (or at least specification) of a defined control interface for the human-computer interaction. Most software-based spatialisation systems provide open interfaces to connect to any sensor or control interface of choice. While this aspect of modularity can provide advantages under certain conditions, we only considered the controller as a fully developed musical instrument, if it incorporates a specific control interface.

Our inventory is based on different sources, which have been analysed systematically in relation to relevant content. These include secondary sources, i.e. textbooks or survey articles on spatialisation practice that have been published since the 1990s. All relevant texts evaluated in our study can be found in Roads (1996), Manning (2013), Brech (2015), Brech and Paland (2015), and Johnson et al. (2014a), along with the primary resources cited therein.

Moreover, we searched the proceedings of relevant international conferences as well as the major journals related to computer music and technology for musical expression, including all years of the New Interfaces for Musical Expression (NIME, 2001-2015), the International Computer Music Conference (ICMC, 1975-2015), 
Table 2 Inventory of spatialisation controllers (in chronological order)

\begin{tabular}{|c|c|c|}
\hline \# & Name of controller/project & Year \\
\hline 1 & Potentiometre relief portico (1951), Pupitre d's space (1952), P. Schaeffer & 1951 \\
\hline 2 & Rotation Mill (Tonmühle), 1959, 1970 for Stockhausen, Expo 1970 in Osaka & 1960 \\
\hline 3 & Photocell mixers $(1967,1968)$, F. Rzewski, D. Behrman & 1966 \\
\hline 4 & Spherical sound controller for German Pavilion, World Expo 1970 (TU Berlin) & 1970 \\
\hline 5 & SAL Mar construction (S. Martirano) & 1971 \\
\hline 6 & Circular relay switch (B. Leitner) & 1971 \\
\hline 7 & HaLaPhon (different versions, 1971-1985) (H.P. Haller, P. Laszlo) & 1971 \\
\hline 8 & Loudspeaker orchestras (Gmebaphone, Acousmonium, BEAST et al.) & 1973 \\
\hline 9 & Hybrid IV (Kobrin 1975) & 1977 \\
\hline 10 & SSSP — a computer-controlled sound distribution system (Federkow et al. 1978) & 1978 \\
\hline 11 & Trails: an interactive system for sound location (Bemardini and Otto 1989) & 1989 \\
\hline 12 & $E I S$ - the expanded instrument system (Oliveros 1991) & 1991 \\
\hline 13 & Data Glove real time control of 3D sound by Gesture (Harada et al. 1992) & 1992 \\
\hline 14 & MusicSpace: a midi-file spatialisation tool (Pachet and Delerue 1999) & 1998 \\
\hline 15 & M2 diffusion - the live diffusion of sound in space, (Moore et al. 2004) & 2004 \\
\hline 16 & Orb3 - adaptive interface for real time diffusion (Livingstone et al. 2005) & 2005 \\
\hline 17 & light-emitting pen controllers (Brown et al. 2005) & 2005 \\
\hline 18 & NAISA spatialization system (Copeland 2014) & 2006 \\
\hline 19 & DJ Spat: spatialized interactions for DJs (Marentakis et al. 2007) & 2007 \\
\hline 20 & multi-touch soundscape renderer (Bredies et al. 2008) & 2008 \\
\hline 21 & Pointing-At Glove and 3D-DJ App, (Torre et al. 2009) & 2009 \\
\hline 22 & Grainsticks, collaborative sound installation (Leslie et al. 2010) & 2010 \\
\hline 23 & Bodycoder system (for V'OCT ritual) (Bokowiec 2011) & 2011 \\
\hline 24 & GAVIP (Gestural auditory visual interactive platform) (Caramiaux et al. 2011) & 2011 \\
\hline 25 & The sound flinger (Carlson et al. 2011) & 2011 \\
\hline 26 & The radiodrum for real time sound spatialization (Ness et al. 2011) & 2011 \\
\hline 27 & WFS gesture control (Fohl and Nogalski 2013) & 2013 \\
\hline 28 & tactile.space (Johnson and Kapur 2013) & 2013 \\
\hline 29 & $S S N$ - sound surfing network (Park et al. 2013) & 2013 \\
\hline 30 & tactile.motion: an iPad-based performance interface (Johnson et al. 2014a, b) & 2014 \\
\hline 31 & Holistic 3D sound controller (Diatkine et al. 2015) & 2015 \\
\hline
\end{tabular}

and the Sound and Music Computing (SMC, 2004-2015), and the Computer Music Journal (1977-2015). In total, our search yielded around three dozen spatialisation instruments (Table 2). ${ }^{11}$

\footnotetext{
${ }^{11}$ The exact figure varies between 31 and 38 depending on the way of counting different versions or parallel developments of basically the same spatialisation instrument. In the following, we will consider the minimal size of the sample for the sake of simplicity.
} 
It should be noted that a couple controllers have been excluded from the sample for various reasons: Some research papers lack a transparent concept of the controller in focus, its mappings or used components; others do not explicitly include a defined controller device, but provide an open interface to connect an arbitrary controller or sensing system.

\subsection{A Classification of Real Time Spatialisation Controllers}

Firstly, we will describe the found manifestations and the general distribution of spatialisation instruments in our classification space. Secondly, we will take a closer look at the clusters and present the individual controllers briefly highlighting their most important specifications. Finally, we will recapitulate and contextualise our findings.

Figure 2 presents the distribution of the collected controllers over the categories defined above. About one third of the controllers include sound generation means, while the majority has been designed as exclusive sound spatialisation device. As expected, the category of instrument-like and augmented controllers is hardly represented in the sample: we could identify only two instruments of this kind. Moreover, controllers providing no sound generating means are not represented in the group of alternate mixed controllers. Most of the controllers for both sound synthesis and spatialisation synthesis can be found in the group of touch controllers that are used to diffuse sound in space. The group of touch controllers with control of sound source position and characteristics generally constitutes the largest portion of the sample (around one quarter of all observed instruments), closely followed by the adjacent group of controllers for sound diffusion.

In the following, we will briefly address the single controllers observed in our study. For more comprehensive information on the respective instruments refer to the given sources.

\subsubsection{Augmented Controllers}

Control of Sound Source Related Parameters One instrument could be classified as augmented controller for sound synthesis and spatial control. DJ Spat was presented by Marentakis et al. (2007) as a spatialisation system to augment the DJ interaction metaphor related to the use of a turntable ${ }^{12}$ during performance. Using motion-tracking sensors and further haptic control elements, the performer was enabled to control the spatial organization of the played sounds as a "bi-product" of

\footnotetext{
${ }^{12}$ Again, the question might arise if this gestural interface can be considered as an augmented instrument linked to the discourse of whether a DJ-turntable represents a musical instrument or not. At this point, we avoid to comment on this topic by using the term augmented controller in reference to a well-established control interface for musical performances.
} 


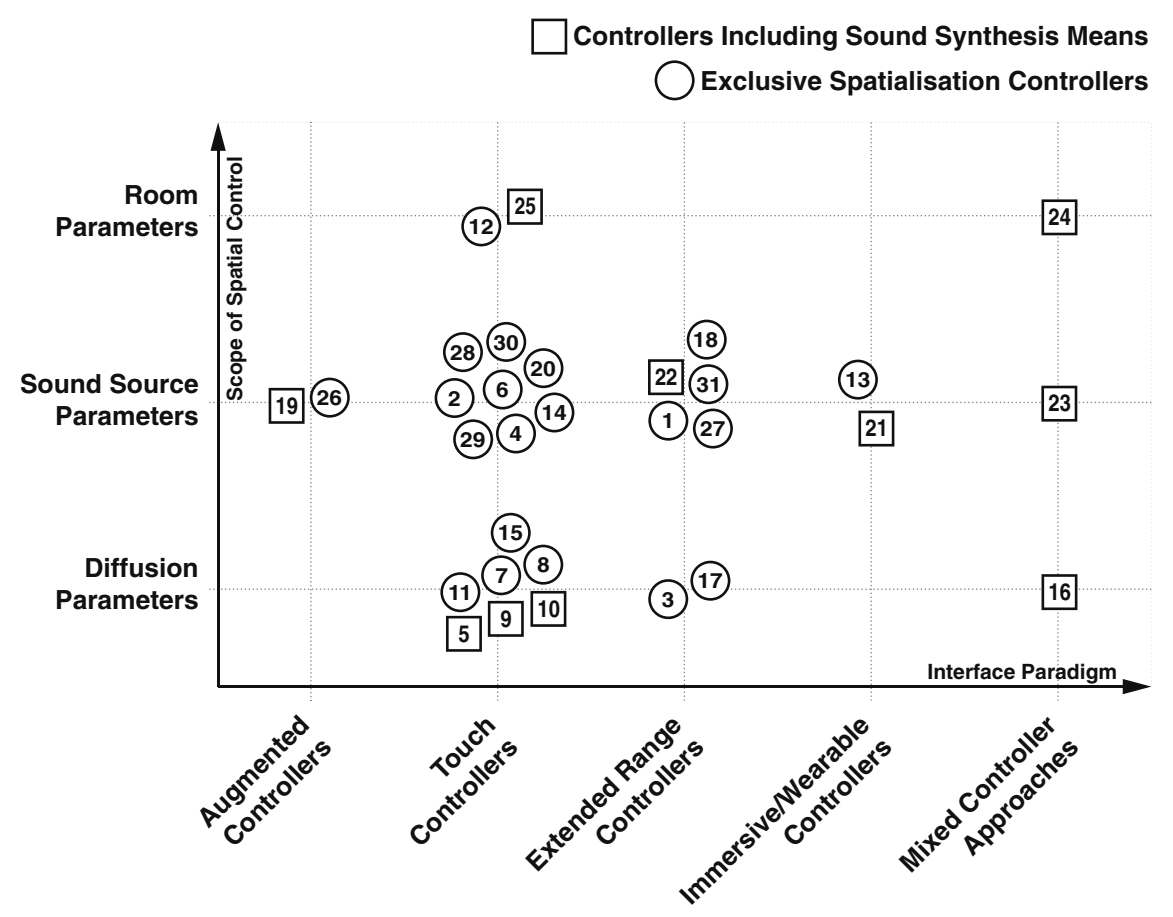

Fig. 2 Classification of real time spatialisation controllers (the numbers in the figure refer to the numbering in Table 2)

his musical performance. The researchers mapped the angular displacement of the performer's hand on the record to the sound source position reproduced through a circular loudspeaker array.

As a counterpart, one augmented controller exclusively for spatialisation was presented by Ness et al. (2011). They developed an approach to use the Radiodrum, a gestural control system from the late 1980s inspired by the playing of a drum with sticks, as a spatialisation system. The authors mapped, among others, controller's position data to the positions of sound sources in space.

\subsubsection{Touch Controllers}

Control of Sound Diffusion Parameters The spatialisation systems from this cluster differ in terms of the used technologies, but show several similarities in relation to the control paradigm. The first three instruments in our list explicitly include control elements for sound synthesis.

The Sal Mar Construction was designed in the early 1970s by composer Salavator Martirano as a real time system for composition and performance of electronic music (Franco 1974). The large electronic instrument consists of 
analogue circuits and digital modules, which allowed the performer to use different sound synthesis techniques. It incorporates a multi-channel matrix system with up to 24 discrete outputs for operations in real time performance. By means of numerous touch-sensitive switches located at the front panel, the sounds could be distributed throughout the performance space.

A similar concept of spatialisation was realized with the Hybrid $\boldsymbol{I V}$ system developed by Edward Kobrin in 1975. It consists, analogously to the hybrid approach by Martirano, of several analogue sound generating and processing components which are controlled by a computer system for composition and real time use (Fedorkow et al. 1978). The multi-channel matrix provides 16 outputs to be routed to a loudspeaker array, likewise by using switches and buttons to assign the signals to the loudspeakers.

The SSSP Sound Distribution System was developed by Fedorkow et al. (1978) in the late 1970s. It was inspired by the general design approach of the two systems described above, but combines a compact and modular design with 16 output-channels and the use of various input devices such as a keyboard and a digitizing tablet. The polyphonic sounds are synthesized by another module of the SSSP system and can be controlled using the same interface.

The following controllers are mainly related to the practice of sound diffusion. While some of them offer sound manipulation means, they are considered as exclusive spatialisation controllers which have been created for the purpose of projecting sound in space.

The HaLaPhon was invented in the late 1960s by Hans-Peter Haller and Peter Laszlo as an instrument to control diffusion and spatialisation in real time performance (Brech and von Coler 2015). The system uses an amplitude panning mechanism to realize virtual (mainly circular) sound movements controlled by switches or automation. Several versions followed making increasingly use of digital technology while maintaining the general concept of the device.

The most important sub-division of instruments in this cluster embraces different kinds of Loudspeaker Orchestras. The Gmebaphone (later known as Cybernephone) was introduced in 1973 (Clozier 2001), one year later the Acousmonium (Brech 2015). Several further developments followed, the BEAST System (Harrison 1999) being one of the most recent and important approaches. While some those systems differ significantly, they all share some fundamental features: They are - to a certain extent - modular in design (for a portable use) and they comprise a fader board based controller assigning the sounds to a multitude of included (and very specific) loudspeakers using amplitude panning methods. These spatialisation controllers can be considered as one crucial aspect of common diffusion practice in electroacoustic music.

Another example for a diffusion system following a similar concept is TRAILS (Tempo Reale Audio Interactive Location System), an interactive controller for sound localization (Bernardini 1989). The matrix-based system was presented in 1988. In contrast to the loudspeaker orchestras mentioned above, it did not explicitly define a particular loudspeaker configuration. 
The M2 system was presented by Mooney et al. (2004) as another modular diffusion system consisting of a sound rendering engine (software running on a computer system) and a specifically designed fader board. The system allows for some specific assignment and grouping functionalities.

Control of Sound Source Related Parameters This cluster of dedicated spatialisation controllers comprises the largest portion of spatialisation instruments in our sample. One of the first spatialisation controllers recorded in literature is the rotation mill (Tonmühle) which was conceptualized already around 1960 at the Technical University in Berlin and later designed for Karlheinz Stockhausen to be used for his spatialisation approach at World Exposition 1970 in Osaka, Japan (Gertich et al. 1996). The functionality of device was very straightforward using a kind of rotational resistance patched to a circle of loudspeakers. By manually turning the crank of the mill, the sound source could be shifted circularly through the audience surrounded by 50 loudspeakers.

A similar control device was realized by Leitner in 1971 as a Circular Relay Switch with a manual crank (Leitner 2016). The sound sources could be distributed throughout the space by using up to 20 loudspeakers dynamically controlled by means of additional encoder knobs.

Another innovative approach was a spherical sound controller, which was also designed for the West German pavilion at the World Expo 1970 by the Electronic Music Studio at the Technical University of Berlin (Brech 2015; Gertich et al. 1996). The spherical controller consisted of 50 sensor buttons, each representing a loudspeaker group in the spherical concert hall. By this means, sound sources could be projected and moved in space.

An example for a different sound spatialisation metaphor was presented with MusicSpace by Pachet and Delerue in 1999. The authors presented a typical approach for considering sound sources as objects, which can be freely localized and moved in the projection space. A standard mixer and object-based sound-rendering engine is used to define positions of sounds or groups of sounds in the performance space.

A different concept of haptic interface was presented by Bredies et al. (2008) referred to as Multi-Touch Soundscape Renderer. The authors use a tabletop device with multi-touch sensing as direct manipulation interface which can be used by multiple users simultaneously. Sound objects represented in the graphical user interface can be manipulated through touch gestures. The object-based sound reproduction is achieved by using wave field synthesis and a circular speaker array.

Tactile.space, showed by Johnson and Kapur (2013), represents a similar interface design approach, which also makes use of a surface-based tabletop interface. Analogously, sound objects' positions can be changed by means of touch gestures, although an amplitude panning method is used to project the sound in space. By presenting tactile.motion, the authors provide an application for mobile tablets to be used as a controller instead of the tabletop interface (Johnson et al. 2014b).

Another innovative mobile spatialisation system was presented by Park et al. (2013). The Sound Surfing Network (SSN) is a system that can be used for smart 
phone-based sound spatialisation. The application is divided into two entities: the performer-app is used to control the object-based sound sources in the performance space, the audience-app turns each smartphone into an element of the loudspeaker array on which the sound is spatialised.

Control of Room Related Parameters We found one device that could be classified as hybrid controller of sound synthesis and spatialisation. The Sound Flinger, presented by Carlson et al. (2011), is an instrument for haptic spatialisation within a quadraphonic sound system. Using four motorized faders, the sounds can be moved around the circle, using intuitive gestures mapped to physical models, which affect both the spatialisation and the sound processing.

Another single spatialisation instrument could be assigned to the category of dedicated spatial controllers: the Expanded Instrument System (EIS), continuously developed since 1963 by Oliveros (1991), is a performance environment that was intended to give the performer control over the acoustic space. This is mainly achieved by means of delay and reverb enhancing the sound image created through amplitude panning. The control interface consists of several foot switches. Multi channel speaker configurations are used to reproduce the extended acoustical scene. Figure 3 illustrates a selection of different controllers for the spatialisation of sound in real time.

\subsubsection{Extended Range Controllers}

Control of Diffusion Parameters The Photocell Mixer (also Photoresistor Mixer) is an early example for an extended range controller exclusively for sound diffusion. It was created in the late 1960s by Frederic Rzewski (1968) and David Behrman (Holmes 2012, 430). Although both composers have developed their mixers independently, the mixers are very similar in design. They consist of panels with several groups of photocells integrated in the signal circuits. Illuminating the photocell of one signal path with a penlight assigns the signal to the one of four outputs and the respective loudspeaker. Thus, the performer diffuses the sound by moving the penlight over the photocells.

Brown et al. (2005) developed an approach to sound diffusion which similarly makes use of hand-held light emitting pen controllers which are tracked by means of a camera system. Referring to the spatial conductor metaphor (Marshall et al. 2007, 232) to decouple diffusion practice from the fader board, gestures performed with the hand-held pen torches are mapped to diffusion parameters of the sound image reproduced by means of the loudspeaker array.

Control of Sound Source Related Parameters Grainstick is the name of a hybrid controller system developed by Leslie and colleagues in 2010. It demonstrates a further approach of multimodal interfaces for a sound field reproduction system by using a combination of infrared motion tracking and accelerometer data of hand-held controllers. Controller data is used for sound synthesis and spatialisation within an elaborated mapping structure. The process can be controlled collaboratively by a group of performers. 

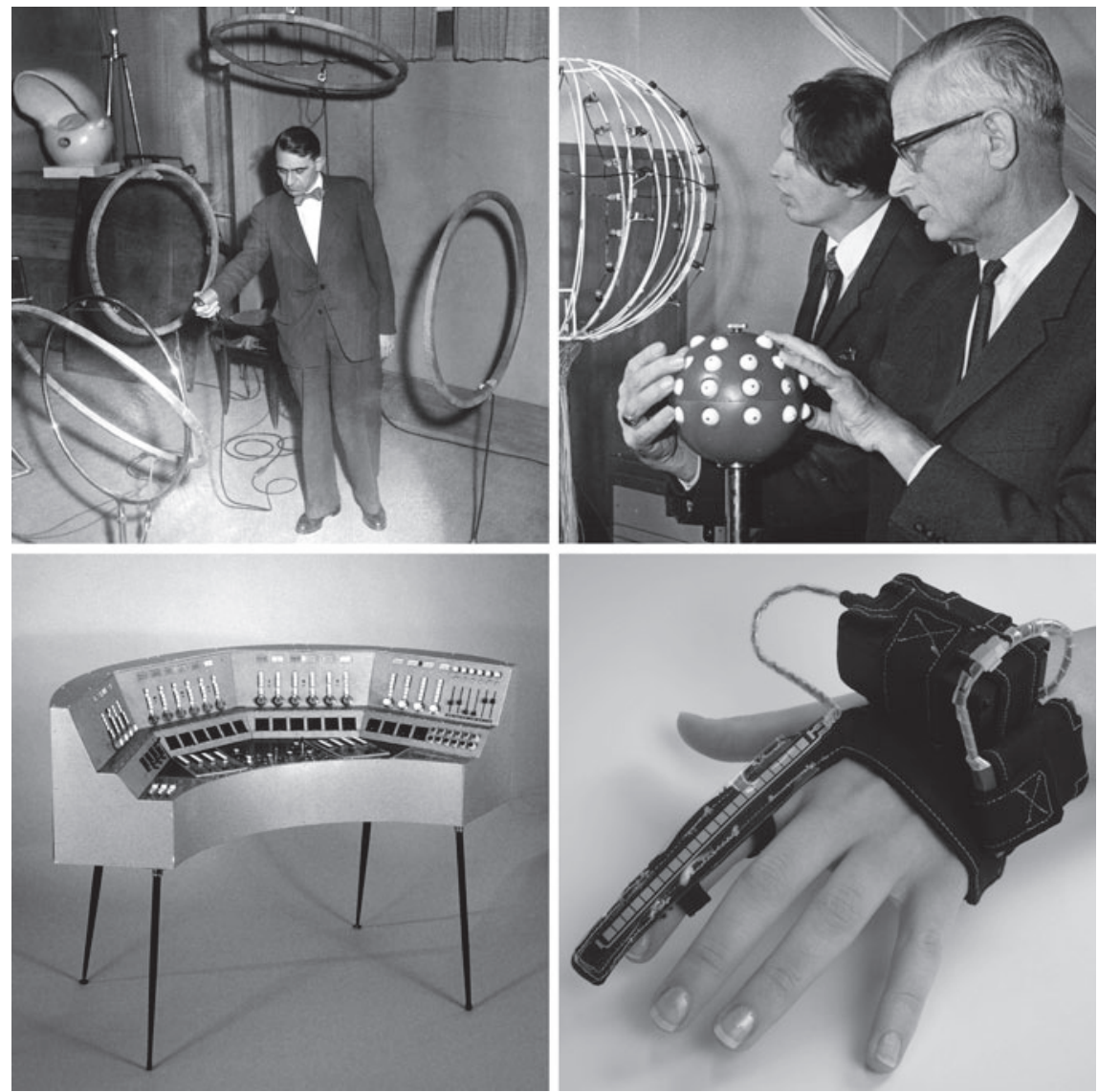

Fig. 3 A selection of controllers for the real-time spatialisation of sound. Top left Pierre Schaeffer playing the Pupitre d'Espace, photo Maurice Lecardent, 1955 (C) INA; top right the spherical spatialisation controller for the World Expo 1970 in Osaka, developed at the Technische Universität Berlin, used by Fritz Winckel, Manfred Krause in the background, photo: TU Archive, 1970 (C) TU Berlin; lower left the controller board of the Gmebaphone2, developed at the GMEB in Bourges, France, photo by MIMO Musical Instrument Museums Online (CC BY-NC-SA); lower right The Pointing-At Glove developed by Giuseppe Torre, photo by courtesy of Cillian O'Sullivan and John McCall

All remaining extended range controllers in this cluster are exclusive sound spatialisation systems. One of the first recorded and most frequently cited spatialisation instruments is the Pupitre d'Espace (space console) presented by Pierre Schaeffer in 1952 (Battier 2015). ${ }^{13}$ The controller worked with four induction coils

\footnotetext{
${ }^{13}$ The pupitre d'espace is a further development of a controller introduced in 1951 as pupitre potentiométrique de relief. The device had the same functionality but worked with controlling three wires which are linked to potentiometers to adjust the signal level send to each loudspeaker (Battier 2015, 127).
} 
mounted around the performer as receiver rings. A further coil was held in the hand of the performer. By moving the coil between the receiver rings, four currents were induced. These controlled the amplifiers sending the signals to the four loudspeakers surrounding the audience space. The speakers were arranged according to the positions of the coils in three spatial axis, thus achieving quasi-periphonic sound spatialisation.

The NAISA Spatialisation system has been developed since 2006 by Copeland (2014) as an interactive performance system for spatial sound projection to up to 24 surrounding loudspeakers. At the core of the gestural spatialisation control is a six degrees-of-freedom motion tracking system with a magnetic sensor connected to the hand of the performer. Gestural movements of the hand are then mapped to the sound sources' positions and orientations. The performer can move quite freely in a certain range around the receiving sensor. The software running on a computer system allows for a very variable use of the system.

Fohl and Nogalski (2013) present another spatialsation system that uses a infrared-camera system for performer's gestural tracking. Their Gesture Controller

for a WFS System approach makes use of markers mounted to the hand of the performer for gesture recognition by means of the camera system. The control over the wave field synthesis renderer is achieved through the mapping of predefined gestures to certain functionality such as positioning, movement patterns or switching of sound sources.

A holistic spatialisation system for multiple sound sources is presented by Diatkine et al. (2015). The researchers use a short-range infrared sensor to track hand gestures and map this data to position data of sound sources, which are reproduced via a higher-order ambisonics and dynamic binaural rendering process. Consequently, the system uses headphones instead of loudspeakers to reproduce the sounds limiting its use to rehearsal contexts rather than to a concert performance.

\subsubsection{Immersive/Wearable Controllers}

Control of Sound Source Related Parameters The Pointing-At Glove was developed by Torre (2013) as a gestural spatialisation controller system incorporating sound generation and manipulation capabilities. The controller glove was realized using a six degrees-of-freedom sensor allowing for the control of periphonic 3D sound spatialisation, including the vertical dimension. Here, the use of the $3 D-D J$ (Torre 2013) application enables the performer to render three-dimensional audio scenes by means of the gestural control data tracked by the glove.

In their conference contribution from 1992 Harada, Sato, Hashimoto and Ohteru present an early design for an immersive, wearable controller system exclusively for spatial sound projection. The authors make use of a Data Glove for the recognition of a performer's gesture. An amplitude panning method controlled by means of midi control data is used to route the sound signals to a 3-dimensional loudspeaker array. 


\subsubsection{Mixed Controller Approaches}

All controllers within this category are hybrid controllers to be used for spatialisation and sound generation.

Control of Diffusion Parameters With Orb3 Livingston and Miranda (2005) present an adaptive sound synthesis and diffusion system. Three mobile sensing globes, which comprise a set of eight different sensors each, collect data within the performance environment. The authors demonstrate an approach of indirect and subconscious user interaction where control data is obtained from several sensors and adaptively mapped to processes controlling the sound diffusion.

Control of Sound Source Related Parameters Another controller design using a set of different controllers was demonstrated by Bokowiec (2011) with his version of a Bodycoder System. The approach is based on a sensor array integrated in a wearable controller system. It mainly consists of two sensing gloves. The system provides motion detection, 12 haptic switches and four bend sensors to control the different functions during performance including sound and video manipulation and the spatialisation through a multichannel loudspeaker array.

Control of Room Related Parameters The last controller in our inventory which is not exclusively designed for sound spatialisation was introduces as $\boldsymbol{G A V I P}$ (Gestural Auditory and Visual Interactive Platform) by Caramiaux and colleagues in 2011. The authors created a virtual space architecture with different means for gestural tracking (camara based and with gyroscope sensors). To achieve inter-modal interaction coherency, the sensor data was mapped to a complex physical model, which then was used to render the 3D audio-visual scene including the synthesis of a virtual dynamic sound field by means of wave field synthesis.

\section{Discussion}

The main objective of this work has been to conduct an inventory of controllers for the real time spatialisation as part of musical performances, and to classify them both along different interface paradigms and along their scope of spatial control. By means of a thorough literature study, we were able to identify 31 different spatialisation interfaces presented in the context of the most relevant conferences and/or mentioned in a selection of important monographs on the subject.

Considering the significance attributed to space as a musical parameter throughout the discourse on electroacoustic music, this seems only a modest number, also compared to the abundance of musical interfaces for sound synthesis, which were presented in the same sources to the same communities. Hence, despite the great interest in concepts and techniques for live-interaction and music, the spatialisation of sound still seems to be regarded as an aspect of musical composition rather than of musical performance. Whether intentionally or not, the classical concept of the performer as sound generator, with the spatial organisation of sound remaining part of the compositional process, is still prevalent. 
The reason for this allocation of roles can hardly be assigned to a lack of technical resources, as it is demonstrated by the 31, quite diverse, approaches described above. Most of them are controllers for spatialisation only, while only a minority (10 out of 31) combines sound synthesis and spatial presentation with one, hybrid interface. Whether this again reflects a traditional role model with respect to the control of sound and space, or whether it reflects practical challenges in interface design and user interaction, is difficult to decide.

Almost all instruments for spatialisation are dedicated to control the position or the movement of individual sound source or of sound sources as a group (diffusion approach), while the control of the (virtual) spatial environment itself is rarely (only with 3 out of 31 tools) addressed.

Concerning the interface paradigm used, the vast majority of existing interfaces are touch controlled, whereas there are only few examples for immersive or wearable spatial controller systems, which are not restricted by operating an immobile apparatus or by a limited spatial range of operation. Taken both aspects together, there seems to be quite a lot of potential for further developments.

The artistic context which most spatialisation controllers were developed for, is quite specific in most cases. Already the early devices presented in the course of the 20th century were custom-built according to the requirements of individual composers and compositions, such as the rotation mill for Stockhausen, Martirano's SalMar Construction, Schaeffer's Pupitre d'Espace, or the loudspeaker orchestras designed by Bayle or the Groupe de Musique Expérimentale des Bourges. Also more recent developments of the last two decades have often been applied to unique artistic projects, and we could not identify a generic control paradigm or a generic interface used for a larger repertoire of music.

The only exception seems to be the fader board paradigm which has become an integral and constitutive part of the sound diffusion practice in the context of acousmatic music and performance practice with loudspeaker orchestras. Although the mixing desk is not only used for the spatialisation there, but also for the assignment of different loudspeaker groups with their individual sound character, this seems the only example where a spatialisation interface is re-used for the performance of different music by different performers. It is no coincidence that this is the only musical area where a notion of performative virtuosity with respect to sound spatialisation could develop.

With this exception, the spatial enactment of sound as performance practice is still characterised by rather individual design solutions. Many of these are modular systems consist of existing interfaces and universal components, such as borrowed sensing and control devices (e.g., gamepads, infrared tracking systems, gyroscopic sensors) which allow performers "(...) to map their performative gestures to any number of musical parameters" (Johnson et al. 2013, 271). The spatial rendering tends to be handled by common spatialisation applications such as Spat, SSR, or Zirkonium, or customised solutions based on common frameworks such as SuperCollider, Max or PureData. Even some vintage spatialisation controllers, formerly built as analogue electronic circuits, have been redeveloped using these software environments, such as Behrman's Photocell Mixer (Behrman 2016; 
Holmes 2012, 430), Oliveros' Expanded Instrument System (Oliveros 2008), or the HaLaPhon recreated as a Max-patch to re-enact pieces by Luigi Nono or Pierre Boulez (Ferguson 2010).

Although such a modular approach offers the shortest and most flexible access to spatial control, the related interfaces will probably not be identified as musical instruments by performers (other than those who developed it) and by the audience. As pointed out by Hardjowirogo (this volume), the establishment and the cultural embeddedness of an interface within a certain aesthetical practice are crucial aspects of instrumentality, which can only be reached by repeated use-not to mention other criteria such as the immediate connection between the performer's actions and the sonic result or the perceived liveness on the side of the audience.

Whether it is desirable to devise a spatial controller as a musical performance instrument, is, of course, a matter of the artistic premises, on which its development is based. Given the structural importance of sound projection in space in current musical practice, however, the creation of tools for its real-time control seems only consequent. If these are supposed to be recognized as musical instruments in a narrow sense, the related technical challenges are still awaiting successful solutions.

\section{References}

Baalman, Marije A. J. (2010). Spatial composition techniques and sound spatialisation technologies. Organised Sound, 15(3), 209-218. doi:10.1017/S1355771810000245

Battier, M. (2015). Recent discoveries in the spatial thought of early musique concrète. In M. Brech \& R. Paland (Eds.), Compositions for audible space. The early electroacoustic music and its contexts. Music and sound culture (pp. 123-36). Columbia: Transcript Verlag.

Behrman, D. (2016). Personal interview with David Behrman in Berlin, May 14, 2016.

Bernardini, N. (1989). Trails: An interactive system for sound location. Ann Arbor, MI: Michigan Publishing, University of Michigan Library.

Birnbaum, D., Fiebrink, R., Malloch, J., \& Wanderley, M. M. (2005). Towards a dimension space for musical devices. In Proceedings of the 2005 Conference on New Interfaces for Musical Expression (pp. 192-95). Singapore: National University of Singapore.

Blauert, J. (1997). Spatial hearing: The psychophysics of human sound localization. Cambridge: MIT press.

Bokowiec, M. A. (2011). VOCT (Ritual): An interactive vocal work for bodycoder system and 8 channel spatialization. In Proceedings of the International Conference on New Interfaces for Musical Expression.

Brech, M. (2015). Der Hörbare Raum Entdeckung, Erforschung Und Musikalische Gestaltung Mit Analoger Technologie. Columbia: Transcript Verlag.

Brech, M., \& Paland, R. (Eds.). (2015). Compositions for audible space. The early electroacoustic music and its contexts. Music and sound culture. Columbia: Transcript Verlag.

Brech, M., \& von Coler, H. (2015). Aspects of space in luigi nono's prometeo and the use of the Halaphon. In M. Brech \& R. Paland (Eds.), Compositions for audible space. The early electroacoustic music and its contexts. Music and sound culture (pp. 193-204). Columbia: Transcript Verlag.

Bredies, K., Alexander Mann, N., Ahrens, J., Geier, M., Spors, S., \& Nischt, M. (2008). The multi-touch sound scape renderer. In Proceedings of the working conference on advanced 
visual interfaces (pp. 466-469). AVI '08. New York, NY, USA: ACM. doi:10.1145/1385569. 1385660

Brown, K., Alcorn, M., \& Rebelo, P. (2005). Sound diffusion using hand-held light-emitting pen controllers. In proceedings of international computer music conference.

Caramiaux, B., Fdili Alaoui, S., Bouchara, T., Parseihian, G., \& Rébillat, M. (2011). Gestural auditory and visual interactive platform. In 14th International conference on digital audio effects (DAFx-11), 69.

Carlson, C., Marschner, E., \& Mccurry, H. (2011). The sound flinger: A haptic spatializer. In Proceedings ICMC 2011.

Chowning, J. M. (1971). The simulation of moving sound sources. Journal of the Audio Engineering Society, 19(1), 2-6.

Clozier, C. (2001). The gmebaphone concept and the cybernéphone instrument. Computer Music Journal, 25(4), 81-90.

Copeland, D. (2014). The NAISA spatialization system. April. http://www.darrencopeland.net/ web2/?page_id $=400$

Diatkine, C., Bertet, S., \& Ortiz, M. (2015). Towards the holistic spatialization of multiple sound sources in 3d, implementation using ambisonics to binaural technique. In Proceedings.

Fedorkow, G., Buxton, W., \& Smith, K. C. (1978). A computer-controlled sound distribution system for the performance of electroacoustic music. Computer Music Journal, 33-42.

Ferguson, P. (2010). Development of a 3D audio panning and realtime visualisation toolset using emerging technologies. Ph.d. thesis, Edinburgh Napier.

Fohl, W., \& Nogalski, M. (2013). A gesture control interface for a wave field synthesis system. In Proceedings of NIME 2013, 341-346.

Franco, S. (1974). Hardware design of a real-time musical system. Champaign: University of Illinois at Urbana-Champaign.

Geier, M., Spors, S., \& Weinzierl, S. (2010). The future of audio reproduction: Technologyformats-applications. In M. Detyniecki, U. Leiner, \& A. Nürnberger (Eds.), Adaptive multimedia retrieval. Identifying, summarizing, and recommending image and music (pp. 117). Springer.

Gertich, F., Gerlach, J., \& Föllmer, G. (1996). Musik, Verwandelt: Das Elektronische Studio Der TU Berlin 1953-1995. Wolke.

Harada, T., Sato, A., Hashimoto, S, \& Ohteru, S. (1992). Real time control of 3D sound space by gesture. In International computer music conference proceedings.

Harrison, J. (1999). Diffusion: Theories and practices, with particular reference to the BEAST system. eContact, 2.

Holmes, T. (2012). Electronic and experimental music: Technology, music, and culture. Routledge.

Jaroszewicz, M. (2015). Compositional strategies in spectral spatialization. California: University of California Riverside.

Johnson, B., \& Kapur, A. (2013). Multi-touch interfaces for phantom source positioning in live sound diffusion. In W. S. Yeo, K. Lee, Alexander Sigman, (Hyunkyung) H. Ji, \& G. Wakefield (Eds.,) 13th international conference on new interfaces for musical expression, NIME 2013, Daejeon, Republic of Korea, May 27-30, 2013 (pp. 213-216). nime.org.

Johnson, B., Murphy, J. W., \& Kapur A. (2013). Designing gestural interfaces for live sound diffusion. In Proceedings of the 39th international computer music conference, ICMC 2013, Perth, Australia, August 12-16, 2013. Michigan Publishing.

Johnson, B., Norris, M., \& Kapur, A. (2014a). Diffusing diffusion: A history of the technological advances in spatial performance. In International computer music conference proceedings 2014.

Johnson, B., Norris, M., \& Kapur, A. (2014b). Tactile.motion: An iPad based performance interface for increased expressivity in diffusion performance. In Music technology meets philosophy - from digital echos to virtual ethos: Joint proceedings of the 40th international computer music conference, ICMC 2014, and the 11th sound and music computing conference, SMC 2014, Athens, Greece, September 14-20, 2014. Michigan Publishing. 
Leitner, B. (1971). Sound architecture-space created through traveling sound. New York: ARTFORUM.

Leitner, B. (2016). Atelier Bernhard Leitner. In Amt der Niederösterreichischen Regierung. Bielefeld: Kerber Verlag.

Leslie, G., Zamborlin, B., Jodlowski, P., \& Schnell, N. (2010). Grainstick: A collaborative, interactive sound installation.

Livingstone, D., \& Miranda, E. (2005). Orb3: Adaptive interface design for real time sound synthesis and diffusion within socially mediated spaces (pp. 65-69). Singapore: National University of Singapore.

Lynch, H., \& Sazdov, R. (2011). An ecologically valid experiment for the comparison of established spatial techniques. In International computer music conference proceedings 2011.

Malham, D. G. (1998). Approaches to spatialisation. Organised Sound, 3(2), 167-177.

Manning, P. (2013). Electronic and computer music. Oxford: Oxford University Press.

Marentakis, G., Peters, N., \& McAdams, S. (2007). DJ spat: Spatialized interactions for DJs. In International computer music conference proceedings 2007.

Marshall, M. T., Malloch, J., \& Wanderley, M. M. (2007). Gesture control of sound spatialization for live musical performance. In M. S. Dias, S. Gibet, M. M. Wanderley, \& R. Bastos (Eds.), Gesture-based human-computer interaction and simulation. Lecture notes in computer science 5085 (pp. 227-38). Berlin: Springer.

Miranda, E., \& Castet, J. (Eds.). (2014). Guide to brain-computer music interfacing. Berlin: Springer.

Miranda, E. R., \& Wanderley, M. M. (2006). New digital musical instruments: Control and interaction beyond the keyboard (Vol. 21). AR Editions, Inc.

Mooney, J. (2005). Sound diffusion systems for the live performance of electroacoustic music. Sheffield: University of Sheffield.

Mooney, J. R., Moore, A., \& Moore, D. (2004). M2 diffusion: The live diffusion of sound in space. In International computer music association.

Mulder, A. (2000). Towards a choice of gestural constraints for instrumental performers. In M. Wanderlay \& M. Battier (Eds.), Trends in gestural control of music (pp. 315-35).

Ness, S., Odowichuk, G., Driessen, P., Tzanetakis, G. (2011). Controlling Real time sound spatialization using the radiodrum. In International computer music conference proceedings 2011.

Oliveros, P. (1991). The expanded instrument system (EIS). In International Computer Music Conference Proceedings 1991.

Oliveros, P. (2008). The expanded instrument system (EIS): An introduction and brief history. Future of Creative Technologies, Journal of the Institute of Creative Technologies, no., 1, 2124.

Overholt, D. (2011). Violin-related HCI: A taxonomy elicited by the musical interface technology design space. In A. L Brooks (Ed.), Arts and technology-second international conference, ArtsIT 2011 (pp. 80-89). Berlin: Springer.

Pachet, F., \& Delerue, O. (1999). Music space: A constraint-based control system for music spatialization. In International Computer Music Conference Proceedings 1999.

Paradiso, J. A. (1997). Electronic music: New ways to play. IEEE Spectrum, 34(12), 18-30. doi:10.1109/6.642965.

Park, S., Ban, S., Hong, D. R., \& Yeo, W. S. (2013). Sound surfing network (SSN): Mobile phone-based sound spatialization with audience collaboration. In Proceedings (pp. 111-14). Korea.

Perez-Lopez, A. (2015). 3DJ: A supercollider framework for real-time sound spatialization. In Proceedings. Graz, Austria.

Peters, N. (2011). Sweet [Re] production: Developing sound spatialization tools for musical applications with emphasis on sweet spot and off-center perception. McGill University.

Peters, N., Lossius, T., Schacher, J., Baltazar, P., Bascou, C., \& Place, T. (2009). A stratified approach for sound spatialization (pp. 219-24). Citeseer. 
Pressing, J. (1990). Cybernetic issues in interactive performance systems. Computer Music Journal, 14(1), 12-25.

Roads, C. (1996). The computer music tutorial. MIT press.

Rzewski, F. (1968). A photoresistor mixer for live performance. Electronic Music Review, 4(4).

Sannicandro, V. (2014). Space and spatialization as discrete parameter in music composition: A space-oriented approach to écriture; from acoustic through informatics to musical notation. Berlin: epubli GmbH, Berlin.

Schacher, J. C. (2007). Gesture control of sounds in 3D space. In Proceedings of the 7th International Conference on New Interfaces for Musical Expression (pp. 358-362). NIME '07. New York, NY, USA: ACM. doi:10.1145/1279740.1279819

Stockhausen, K. (1959). Musik Im Raum. Die Reihe, Berichte-Analyse, 5, 67-72.

Torre, G., Sazdov, R., \& Konczewska, D. (2009). MOLITVA-Composition for Voice, Live Electronics, Pointing-At Glove Device and 3-D Setup of Speakers. Proceedings of the International Conference on New Interfaces for Musical Expression (NIME), 330.

Torre, G. (2013). The design of a new musical glove: A live performance approach. Limerick: University of Limerick.

Valiquet, P. (2011). The spatialisation of stereophony: Taking positions in post-war electroacoustic music. In International computer music conference proceedings 2011.

Wanderley, M. M., \& Orio, N. (2002). Evaluation of input devices for musical expression: Borrowing tools from Hci. Computer Music Journal, 26(3), 62-76.

Xenakis, I. (1992). Formalized music: Thought and mathematics in music, revised edition. Stuyvesant.

Zvonar, R. (2000). An extremely brief history of spatial music in the 20th century. Surround Professional Magazine. 